\title{
Test de atención D2: Datos normativos y desarrollo evolutivo de la atención en educación primaria
}

\author{
Juan E. Jiménez ${ }^{1}$, Sergio Hernández ${ }^{1}$, Eduardo García ${ }^{1}$, Alicia Díaz ${ }^{2}$, \\ Cristina Rodríguez ${ }^{1}$ y Raquel Martín ${ }^{1}$ \\ ${ }^{1}$ Universidad de La Laguna (España) \\ ${ }^{2}$ Universidad de Las Palmas de Gran Canaria (España)
}

El objetivo de este trabajo ha sido analizar el desarrollo evolutivo de la atención y obtener datos normativos de la prueba de atención d2 en escolares de Educación Primaria (EP). Con este propósito se ha seleccionado una muestra de 1.032 alumnos escolarizados desde primero hasta sexto curso de EP en la Comunidad Autónoma de Canarias, con edades comprendidas entre los 6 años y 3 meses y los 12 años y 4 meses. En cuanto a la variable edad se analizaron los cambios que se observan con el paso de los años en las variables que mide el d2. Los resultados encontrados ponen de manifiesto la existencia de cambios significativos en las variables de productividad y efectividad, y no así en la medida de errores. Asimismo, se han calculado los percentiles correspondientes a cada una de las medidas del d2 para cada rango de edad.

Palabras clave: Atención, desarrollo evolutivo, evaluación, baremación, Test d2.

Attention test D2: Normative data and attention development in elementary grades. The main purpose of this study has been to analyze the attention development in children of elementary grades and to obtain standardization of the test d2. A sample of 1.032 was selected $1^{\text {st }}$ to $6^{\text {th }}$ grade belonging to schools in the Canary Islands with an age range between 6 years and 3 months old to 12 years and 4 months old. We analyzed changes observed over the years in all variables measured by Test $\mathrm{d} 2$. The results found reveal the existence of significant changes in the productivity variables and effectiveness but not in the measure of errors. Furthermore, the percentiles were calculated for each of the measures of $\mathrm{d} 2$ for each age range.

Key words: Attention, development, assessment, standardization, Test $\mathrm{d} 2$.

\footnotetext{
Correspondencia: Juan E. Jiménez. Departamento de Psicología Evolutiva y de la Educación. Universidad de La Laguna. Campus de Guajara, C.P. 38200. Islas Canarias (España). E-mail: ejimenez@ull.es
} 
La atención ha sido definida como el mecanismo implicado directamente en la activación y el funcionamiento de los procesos de selección, distribución y mantenimiento de la actividad psicológica (García, 2008). Se trata, por tanto, de un proceso complejo que al estar constituida por distintos subprocesos ha dado lugar a establecer distintas clasificaciones y subtipos asociados también a diferentes mecanismos o circuitos neurales (Cuesta et al., 2007).

Las alteraciones atencionales están presentes en determinados trastornos como la negligencia unilateral, el síndrome de Balint y la prosopagnosia o ceguera facial (Castillo, 2009). Asimismo, las alteraciones atencionales están asociadas a diferentes enfermedades como las demencias, epilepsia y a trastornos del desarrollo como el autismo o el trastorno por déficit de atención con o sin hiperactividad (TDAH) (Miranda, García y Soriano, 2005). Como ya ha sido descrito en la presentación de esta monografía, el TDAH es definido como un trastorno del desarrollo caracterizado por unos niveles evolutivamente inapropiados de problemas atencionales, hiperactividad e impulsividad (Barkley, 2006). En el trabajo que aquí presentamos nos centramos en la atención considerada ésta como una función independiente y diferenciada de las funciones ejecutivas (FE), aunque se ha llegado a postular que las alteraciones atencionales podrían ser un síntoma de alteración del funcionamiento ejecutivo (Rebollo y Montiel, 2006). Veamos, por tanto, los distintos procesos implicados en esta actividad psicológica.

La atención selectiva puede definirse como la capacidad para centrarse en uno o dos estímulos importantes, mientras se suprime deliberadamente la consciencia de otros estímulos distractores (Zillmer y Spiers, 1998). Se encarga de controlar los procesos y mecanismos por los cuales el organismo procesa tan sólo una parte de toda la información, y da respuestas tan sólo a aquellas demandas del ambiente que son realmente útiles o importantes para el individuo. En este sentido, la atención selectiva implica la selección de los estímulos que se presentan en el ambiente, y la selección del proceso y/o respuesta que se va a realizar. La atención dividida hace referencia a la actividad mediante la cual se ponen en marcha los mecanismos que el organismo utiliza para dar respuesta ante las múltiples demandas del ambiente. En este caso se trata no de seleccionar aspectos específicos de la información, sino de atender a todo lo que se pueda al mismo tiempo. Y la atención sostenida ha sido definida como la actividad que pone en marcha los procesos y/o mecanismos por los cuales el organismo es capaz de mantener el foco atencional y permanecer alerta ante la presencia de determinados estímulos durante períodos de tiempo relativamente largos (Parasuraman, 1984).

Todos estos aspectos diferentes de la atención están regulados por estructuras cerebrales distintas que tienen periodos de neurodesarrollo diferentes (Rebollo y Montiel, 2006). Así, por ejemplo, como han señalado estos mismos autores la atención más elemental o involuntaria depende del funcionamiento del Sistema Reticular 
Ascendente (SAR) proyectado en toda la corteza cerebral desde el tronco cerebral, de naturaleza noradrenérgica y que se desarrolla precozmente en el primer año de vida. La atención selectiva y la atención sostenida están reguladas por estructuras corticales y subcorticales. Entre las estructuras corticales está la corteza prefrontal y las cortezas sensoriales. Y, en el caso de las estructuras subcorticales, el tálamo óptico, el cuerpo estriado (núcleo caudado y lenticular), los núcleos septales y de Meynert y el cerebelo.

Veamos de forma más detallada cómo estas estructuras cerebrales juegan un papel importante en los procesos selectivos de la atención, ya que el presente trabajo se centra más directamente en este tipo de procesos. En el caso del tálamo se ha demostrado que existen neuronas que sólo responden a los estímulos novedosos y que se habitúan rápidamente (Jasper y Bertrand, 1966). Por otro lado, si se estimula eléctricamente el hipocampo se producen respuestas de orientación y conductas de exploración, y esta estructura estaría implicada en la selección estimular como sería la supresión de la información de los estímulos irrelevantes. También Posner y Petersen (1990) demostraron mediante el uso de la técnica de cuantificación de imágenes de Tomografía por Emisión de Positrones (TEP), que la actividad del núcleo pulvinar se relaciona con los procesos selectivos, y en otros estudios se ha observado que una lesión en esta zona provoca dificultades a la hora de dirigir la atención (Rafal y Posner, 1987). A esto habría que añadir el papel que juega la corteza prefrontal y ciertas áreas como sería el lóbulo prefrontal y el parietal. Respecto a la primera habría que señalar que juega un papel fundamental en el control voluntario de la atención, y prueba de ello es que lesiones en esta estructura traen consigo alteraciones de la atención voluntaria y de distracción (Luria, 1984). Y, respecto al lóbulo parietal, se ha demostrado que lesiones en esta área producen déficits de atención selectiva. Así, por ejemplo, lesiones en la corteza parietal inferior se asocian con déficits de atención selectiva como es el síndrome de desatención unilateral, y en el caso de la corteza parietal posterior con los procesos de desenganche de la atención.

\section{Desarrollo evolutivo de la atención e hipótesis explicativas}

Si bien hemos descrito que todos estos aspectos diferentes de la atención se vinculan a determinadas estructuras cerebrales que se desarrollan en diferentes momentos de la vida, existe un debate acerca de si se puede hablar o no de un desarrollo atencional en términos estrictos (intradominio), o por el contrario de un desarrollo cognitivo general (interdominio), dentro del cual estaría como un componente más la atención (Vega, 1984). García (2008) nos ofrece una revisión de estas teorías agrupándolas del siguiente modo. Por un lado, tendríamos aquellas teorías que consideran que existe un desarrollo progresivo de los distintos mecanismos atencionales que es independiente del desarrollo de los restantes procesos psicológicos. En este caso se considera que durante la infancia existe una mayor tendencia a la distraibilidad y un 
menor control atencional en comparación a la vida adulta. Un segundo grupo parte de que el desarrollo atencional depende del desarrollo de otros procesos cognitivos, y en este sentido se acentúa el vínculo existente entre percepción y atención y se considera que ésta es una propiedad selectiva de la percepción. Y, por último, un tercer grupo de teorías sostienen que el desarrollo de la atención va unido al desarrollo cognitivo general, es decir, al desarrollo de otros procesos psicológicos, en este caso no solo a la percepción sino también a la memoria, el pensamiento y a la inteligencia. Solo se considera que el control atencional se desarrolla de forma más específica e independiente de los restantes procesos cognitivos. Por tanto, el rendimiento obtenido por los niños en las pruebas de atención se entiende que depende tanto del desarrollo cognitivo alcanzado como por el nivel de desarrollo del autocontrol atencional.

En el presente estudio hemos abordado un doble objetivo. Por una parte, analizar el desarrollo evolutivo de la atención en población escolar de educación primaria, a partir de las medidas y parámetros que establece el Test de atención d2. Y por otra, obtener datos normativos para la población escolar canaria de cada uno de los parámetros que se incluyen en esta prueba.

\section{MÉTODO}

\section{Participantes}

Se seleccionó una muestra de 1.032 niños canarios de edades comprendidas entre los 6 años y 3 meses y los 12 años y 4 meses. Los escolares seleccionados tenían el español como primera lengua, no presentaban daño neurológico, psiquiátrico o dificultades de aprendizaje y no formaban parte del estadillo de Educación Especial. La participación del alumnado en la administración de la prueba se hizo de manera voluntaria. En la tabla 1 se recoge la distribución de la muestra según rango de edad y sexo.

Tabla 1. Distribución de la muestra según rango de edad y sexo

\begin{tabular}{lrrr}
\hline \multirow{2}{*}{ EDAD } & \multicolumn{2}{c}{ Sexo } & \multicolumn{1}{c}{ Total } \\
\cline { 2 - 5 } & \multicolumn{1}{c}{ Varón } & \multicolumn{1}{c}{ Mujer } & \multicolumn{1}{c}{ Varón } \\
\hline 6 años y 3 meses -7 años y 4 meses & 101 & 103 & 204 \\
\hline 7 años y 5 meses - 8 años y 4 meses & 87 & 80 & 167 \\
\hline 8 años y 5 meses - 9 años y 4 meses & 90 & 89 & 179 \\
\hline 9 años y 5 meses - 10 años y 4 meses & 84 & 72 & 156 \\
\hline 10 años y 5 meses - 11 años y 4 meses & 66 & 98 & 164 \\
\hline 11 años y 5 meses - 12 años y 4 meses & 82 & 80 & 162 \\
\hline Total & 510 & 522 & 1032 \\
\hline
\end{tabular}

\section{Instrumentos}

Test de Atención d2 (Brickenkamp, 2002). Es un test de tiempo limitado para medir la atención selectiva y la concentración mental, entendida como la capacidad de atender selectivamente a ciertos aspectos relevantes de una tarea mientras se ignoran los 
irrelevantes (v.gr., realizar una búsqueda selectiva) y, además, hacerlo de forma rápida y precisa. El test se puede administrar tanto de forma individual como colectiva con una duración entre 8 y 10 minutos. Existe un total de 14 líneas con 47 caracteres, es decir, por un total de 658 elementos; estos estímulos contienen las letras "d" o "p" que pueden estar acompañados de una o dos pequeñas líneas situadas, individualmente o en pareja, en la parte superior o inferior de cada letra. La tarea del sujeto consiste en revisar atentamente, de izquierda a derecha, el contenido de cada línea y marcar toda letra "d" que tenga dos pequeñas rayitas (las dos arriba, las dos debajo o una arriba y otra debajo). En el Manual estos elementos (es decir, los estímulos correctos) se conocen como elementos relevantes. Las demás combinaciones (las "p" con o sin rayitas y las "d" con o ninguna rayita) se consideran "irrelevantes" y no deberían ser marcadas. En cada línea el sujeto dispone de 20 segundos. Las puntuaciones resultantes son: TR, total de respuestas, números de elementos intentados en las 14 líneas; TA, total de aciertos, número de elementos relevantes correctos; $\mathrm{O}$, omisiones, número de elementos relevantes intentados pero no marcados; $\mathrm{C}$, comisiones, número de elementos irrelevantes marcados; TOT, efectividad total en la prueba, es decir, TR - $(\mathrm{O}+\mathrm{C})$; CON, índice de concentración o TA-C; TR+, línea con mayor número de elementos intentados; TR-, línea con menor número de elementos intentados y VAR, índice de variación o diferencia (TR+)-(TR-).

\section{Diseño y procedimiento}

Utilizamos un diseño transversal donde la variable independiente fue la Edad (6 niveles, 6.3-7.4; 7.5-8.4; 8.5-9.4; 9.5-10.4; 10.5-11.4 y 11.5-12.4 años, meses) y como variable dependiente las diferentes medidas del d2. La evaluación fue llevada a cabo por Psicólogas previamente entrenadas. Las evaluaciones se realizaron en un aula suficientemente iluminada y alejada de ruidos y distracciones del centro escolar al que asistía el alumno seleccionado.

\section{RESULTADOS}

Inicialmente comprobamos que la distribución de la muestra en función de la variable sexo en cada grupo de edad era similar $\left[X^{2}(5)=7.37 ; p=0.19\right]$. Las medias y desviaciones típicas obtenidas para cada una de las medidas del d2 en cada grupo de edad se muestran en la tabla 2.

\section{A. Perfil evolutivo (análisis de tendencia)}

Con el objetivo de estudiar si se producen cambios con el paso de los años en cada una de las medidas del d2, llevamos a cabo un ANOVA de un factor tomando la variable edad como variable de agrupamiento y las medidas del d2 como variable dependiente. A su vez se llevó a cabo un análisis de tendencia, y debido a la disparidad del tamaño de los grupos se consultó la solución ponderada. 
JIMÉNEZ et al. Test de atención D2: datos normativos y desarrollo evolutivo

Tabla 2. Medias y desviaciones típicas en las medidas del Test d2 según rango de edad y sexo

\begin{tabular}{|c|c|c|c|c|c|c|c|c|c|c|}
\hline EDAD & SEXO & & TR & TA & $\mathrm{O}$ & $\mathrm{C}$ & TR+ & TR- & $\mathrm{CON}$ & VAR \\
\hline \multirow{12}{*}{$\begin{array}{l}6 \text { años y } 3 \\
\text { meses }-7 \text { años y } \\
4 \text { meses }\end{array}$} & \multirow{4}{*}{$\mathrm{H}$} & $\mathrm{M}$ & 195.95 & 75.38 & 8.60 & 6.32 & 22.43 & 7.57 & 69.46 & 14.86 \\
\hline & & $\mathrm{N}$ & 98.00 & 98.00 & 98.00 & 97.00 & 98.00 & 98.00 & 97.00 & 98.00 \\
\hline & & D T & 52.53 & 23.51 & 15.04 & 9.90 & 8.61 & 4.44 & 26.53 & 9.30 \\
\hline & & $\mathrm{R}$ & 360.00 & 138.00 & 98.00 & 62.00 & 47.00 & 19.00 & 133.00 & 45.00 \\
\hline & \multirow{4}{*}{ M } & $\mathrm{M}$ & 193.23 & 73.17 & 8.62 & 12.10 & 22.14 & 6.57 & 62.86 & 15.58 \\
\hline & & $\mathrm{N}$ & 101.00 & 102.00 & 102.00 & 98.00 & 101.00 & 100.00 & 98.00 & 100.00 \\
\hline & & D T & 59.34 & 28.22 & 11.52 & 20.04 & 7.76 & 4.25 & 25.98 & 7.74 \\
\hline & & $\mathrm{R}$ & 374.00 & 173.00 & 62.00 & 100.00 & 38.00 & 26.00 & 120.00 & 40.00 \\
\hline & \multirow{4}{*}{$\mathrm{T}$} & $\mathrm{M}$ & 194.57 & 74.25 & 8.61 & 9.23 & 22.28 & 7.07 & 66.14 & 15.22 \\
\hline & & $\mathrm{N}$ & 199.00 & 200.00 & 200.00 & 195.00 & 199.00 & 198.00 & 195.00 & 198.00 \\
\hline & & D T & 55.97 & 25.98 & 13.33 & 16.05 & 8.17 & 4.36 & 26.39 & 8.53 \\
\hline & & $\mathrm{R}$ & 430.00 & 173.00 & 98.00 & 100.00 & 47.00 & 26.00 & 133.00 & 45.00 \\
\hline \multirow{12}{*}{$\begin{array}{l}7 \text { años y } 5 \\
\text { meses }-8 \text { años y } \\
4 \text { meses }\end{array}$} & \multirow{4}{*}{$\mathrm{H}$} & $\mathrm{M}$ & 230.75 & 91.79 & 6.45 & 6.99 & 24.41 & 9.84 & 86.84 & 14.58 \\
\hline & & $\mathrm{N}$ & 84.00 & 84.00 & 85.00 & 83.00 & 85.00 & 85.00 & 82.00 & 85.00 \\
\hline & & D T & 57.85 & 23.93 & 11.70 & 11.82 & 7.30 & 5.05 & 24.99 & 8.48 \\
\hline & & $\mathrm{R}$ & 349.00 & 122.00 & 66.00 & 99.00 & 34.00 & 19.00 & 118.00 & 43.00 \\
\hline & \multirow{4}{*}{ M } & $\mathrm{M}$ & 229.57 & 90.39 & 7.16 & 7.72 & 23.33 & 10.34 & 82.67 & 12.99 \\
\hline & & $\mathrm{N}$ & 79.00 & 79.00 & 79.00 & 79.00 & 79.00 & 79.00 & 79.00 & 79.00 \\
\hline & & D T & 65.64 & 28.23 & 15.42 & 13.99 & 6.74 & 5.47 & 32.53 & 6.39 \\
\hline & & $\mathrm{R}$ & 344.00 & 147.00 & 126.00 & 83.00 & 39.00 & 26.00 & 152.00 & 40.00 \\
\hline & \multirow{4}{*}{$\mathrm{T}$} & $\mathrm{M}$ & 230.18 & 91.11 & 6.79 & 7.35 & 23.89 & 10.08 & 84.80 & 13.81 \\
\hline & & $\mathrm{N}$ & 163.00 & 163.00 & 164.00 & 162.00 & 164.00 & 164.00 & 161.00 & 164.00 \\
\hline & & D T & 61.56 & 26.03 & 13.58 & 12.89 & 7.04 & 5.24 & 28.92 & 7.57 \\
\hline & & $\mathrm{R}$ & 367.00 & 147.00 & 126.00 & 99.00 & 39.00 & 26.00 & 152.00 & 53.00 \\
\hline \multirow{12}{*}{$\begin{array}{l}8 \text { años y } 5 \\
\text { meses }-9 \text { años y } \\
4 \text { meses }\end{array}$} & \multirow{4}{*}{$\mathrm{H}$} & $\mathrm{M}$ & 256.12 & 103.02 & 6.90 & 8.00 & 25.34 & 11.87 & 95.02 & 13.47 \\
\hline & & $\mathrm{N}$ & 89.00 & 89.00 & 89.00 & 89.00 & 89.00 & 89.00 & 89.00 & 89.00 \\
\hline & & D T & 51.89 & 22.02 & 10.74 & 12.68 & 6.42 & 4.64 & 27.92 & 6.51 \\
\hline & & $\mathrm{R}$ & 283.00 & 118.00 & 61.00 & 85.00 & 33.00 & 24.00 & 164.00 & 37.00 \\
\hline & \multirow{4}{*}{ M } & $\mathrm{M}$ & 266.62 & 103.96 & 9.90 & 6.73 & 26.52 & 12.25 & 98.00 & 14.27 \\
\hline & & $\mathrm{N}$ & 89.00 & 89.00 & 89.00 & 88.00 & 89.00 & 89.00 & 88.00 & 89.00 \\
\hline & & D T & 52.86 & 24.63 & 14.58 & 14.40 & 7.61 & 4.67 & 24.10 & 8.64 \\
\hline & & $\mathrm{R}$ & 344.00 & 167.00 & 87.00 & 110.00 & 38.00 & 23.00 & 122.00 & 50.00 \\
\hline & \multirow{4}{*}{$\mathrm{T}$} & $\mathrm{M}$ & 261.37 & 103.49 & 8.40 & 7.37 & 25.93 & 12.06 & 96.50 & 13.87 \\
\hline & & $\mathrm{N}$ & 178.00 & 178.00 & 178.00 & 177.00 & 178.00 & 178.00 & 177.00 & 178.00 \\
\hline & & D T & 52.49 & 23.30 & 12.85 & 13.54 & 7.05 & 4.64 & 26.06 & 7.64 \\
\hline & & $\mathrm{R}$ & 359.00 & 167.00 & 87.00 & 110.00 & 38.00 & 24.00 & 164.00 & 54.00 \\
\hline \multirow{12}{*}{$\begin{array}{l}9 \text { años y } 5 \\
\text { meses - } 10 \text { años } \\
\text { y } 4 \text { meses }\end{array}$} & & $\mathrm{M}$ & 284,33 & 114,29 & 7,26 & 4,89 & 27,62 & 14,15 & 110,18 & 13.46 \\
\hline & $\mathrm{H}$ & $\mathrm{N}$ & 84.00 & 84.00 & 84.00 & 83.00 & 84.00 & 84.00 & 83.00 & 84.00 \\
\hline & H & D T & 47.44 & 21.94 & 12.13 & 5.41 & 6.56 & 4.43 & 22.54 & 7.27 \\
\hline & & $\mathrm{R}$ & 241.00 & 116.00 & 81.00 & 30.00 & 33.00 & 26.00 & 113.00 & 35.00 \\
\hline & & $\mathrm{M}$ & 279.94 & 111.66 & 6.45 & 3.93 & 27.93 & 13.23 & 107.73 & 14.70 \\
\hline & $M$ & $\mathrm{~N}$ & 71.00 & 71.00 & 71.00 & 71.00 & 71.00 & 71.00 & 71.00 & 71.00 \\
\hline & $\mathbf{M}$ & $\mathrm{DT}$ & 61.57 & 24.64 & 9.37 & 5.25 & 6.62 & 5.02 & 26.32 & 7.31 \\
\hline & & $\mathrm{R}$ & 362.00 & 135.00 & 52.00 & 30.00 & 32.00 & 28.00 & 134.00 & 52.00 \\
\hline & & $\mathrm{M}$ & 282.32 & 113.08 & 6.89 & 4.45 & 27.76 & 13.73 & 109.05 & 14.03 \\
\hline & $\mathrm{T}$ & $\mathrm{N}$ & 155.00 & 155.00 & 155.00 & 154.00 & 155.00 & 155.00 & 154.00 & 155.00 \\
\hline & 1 & D T & 54.23 & 23.18 & 10.93 & 5.34 & 6.57 & 4.71 & 24.30 & 7.29 \\
\hline & & $\mathrm{R}$ & 362.00 & 135.00 & 81.00 & 30.00 & 35.00 & 30.00 & 134.00 & 56.00 \\
\hline & & $\mathrm{M}$ & 308.53 & 125.40 & 6.81 & 3.81 & 28.48 & 15.58 & 121.56 & 12.91 \\
\hline & $\mathrm{H}$ & $\mathrm{N}$ & 64.00 & 63.00 & 64.00 & 64.00 & 64.00 & 64.00 & 63.00 & 64.00 \\
\hline & $\mathrm{H}$ & $\mathrm{DT}$ & 60.76 & 24.17 & 11.72 & 4.89 & 5.68 & 4.31 & 26.07 & 4.62 \\
\hline & & $\mathrm{R}$ & 227.00 & 93.00 & 66.00 & 26.00 & 28.00 & 19.00 & 119.00 & 26.00 \\
\hline & & $\mathrm{M}$ & 323.80 & 129.46 & 7.55 & 5.05 & 29.62 & 16.47 & 124.41 & 12.90 \\
\hline 10 años y 5 & $M$ & $\mathrm{~N}$ & 98.00 & 98.00 & 98.00 & 98.00 & 98.00 & 97.00 & 98.00 & 98.00 \\
\hline $\begin{array}{l}\text { meses - } 11 \text { anos } \\
\text { y } 4 \text { meses }\end{array}$ & M & $\mathrm{DT}$ & 59.54 & 24.48 & 11.47 & 13.95 & 5.64 & 5.44 & 24.38 & 6.18 \\
\hline & & $\mathrm{R}$ & 386.00 & 134.00 & 79.00 & 101.00 & 31.00 & 32.00 & 131.00 & 54.00 \\
\hline & & $\mathrm{M}$ & 317.77 & 127.87 & 7.26 & 4.56 & 29.17 & 16.12 & 123.29 & 12.90 \\
\hline & $\mathrm{T}$ & $\mathrm{N}$ & 162.00 & 161.00 & 162.00 & 162.00 & 162.00 & 161.00 & 161.00 & 162.00 \\
\hline & $\mathrm{T}$ & D T & 60.30 & 24.37 & 11.54 & 11.27 & 5.66 & 5.02 & 25.02 & 5.60 \\
\hline & & $\mathrm{R}$ & 386.00 & 134.00 & 79.00 & 101.00 & 31.00 & 32.00 & 131.00 & 54.00 \\
\hline
\end{tabular}

Nota. H: Hombre, M: Mujer, T: Total, R: Rango, TR: total de respuestas, TA: total de aciertos, O: omisiones, C: comisiones, CON: índice de concentración, TR+: línea con mayor $n^{\circ}$ de elementos intentados, TR-: línea con menor $\mathrm{n}^{\mathrm{o}}$ de elementos intentados, VAR: índice de variación o diferencia (TR+)-(TR-). 
Tabla 2. Medias y desviaciones típicas en las medidas del Test d2 según rango de edad y sexo (Continuación)

\begin{tabular}{|c|c|c|c|c|c|c|c|c|c|c|}
\hline EDAD & SEXO & & TR & $\mathrm{TA}$ & $\mathrm{O}$ & $\mathrm{C}$ & TR+ & TR- & CON & VAR \\
\hline \multirow{12}{*}{$\begin{array}{l}11 \text { años y } 5 \\
\text { meses - } 12 \\
\text { años y } 4 \\
\text { meses }\end{array}$} & \multirow{4}{*}{$\mathrm{H}$} & M & 326.83 & 130.73 & 7.29 & 4.46 & 30.44 & 16.53 & 126.26 & 13.91 \\
\hline & & $\mathrm{N}$ & 80.00 & 80.00 & 80.00 & 80.00 & 80.00 & 80.00 & 80.00 & 80.00 \\
\hline & & $\mathrm{DT}$ & 51.68 & 19.83 & 8.93 & 12.40 & 5.17 & 4.31 & 23.62 & 4.91 \\
\hline & & $\mathrm{R}$ & 265.00 & 116.00 & 53.00 & 108.00 & 28.00 & 23.00 & 148.00 & 23.00 \\
\hline & \multirow{4}{*}{ M } & $\mathrm{M}$ & 336.70 & 128.78 & 13.78 & 4.05 & 31.80 & 16.64 & 126.06 & 15.16 \\
\hline & & $\mathrm{N}$ & 80.00 & 80.00 & 80.00 & 78.00 & 80.00 & 80.00 & 78.00 & 80.00 \\
\hline & & D T & 68.92 & 25.80 & 23.52 & 8.02 & 7.93 & 4.96 & 27.90 & 7.20 \\
\hline & & $\mathrm{R}$ & 293.00 & 120.00 & 139.00 & 59.00 & 31.00 & 23.00 & 154.00 & 31.00 \\
\hline & \multirow{4}{*}{$\mathrm{T}$} & $\mathrm{M}$ & 331.76 & 129.75 & 10.53 & 4.26 & 31.12 & 16.58 & 126.16 & 14.54 \\
\hline & & $\mathrm{N}$ & 160.00 & 160.00 & 160.00 & 158.00 & 160.00 & 160.00 & 158.00 & 160.00 \\
\hline & & D T & 60.92 & 22.96 & 18.03 & 10.44 & 6.71 & 4.63 & 25.74 & 6.18 \\
\hline & & $\mathrm{R}$ & 301.00 & 120.00 & 139.00 & 108.00 & 31.00 & 24.00 & 154.00 & 31.00 \\
\hline \multirow{12}{*}{ Total } & \multirow{4}{*}{$\mathrm{H}$} & M & 262.84 & 104.87 & 7.27 & 5.87 & 26.21 & 12.28 & 99.64 & 13.93 \\
\hline & & $\mathrm{N}$ & 499.00 & 498.00 & 500.00 & 496.00 & 500.00 & 500.00 & 494.00 & 500.00 \\
\hline & & $\mathrm{DT}$ & 70.02 & 29.70 & 11.93 & 10.26 & 7.31 & 5.54 & 32.30 & 7.22 \\
\hline & & $\mathrm{R}$ & 456.00 & 187.00 & 98.00 & 108.00 & 47.00 & 27.00 & 183.00 & 47.00 \\
\hline & \multirow{4}{*}{ M } & $\mathrm{M}$ & 270.13 & 105.54 & 8.91 & 6.79 & 26.77 & 12.47 & 99.59 & 14.26 \\
\hline & & $\mathrm{N}$ & 518.00 & 519.00 & 519.00 & 512.00 & 518.00 & 516.00 & 512.00 & 517.00 \\
\hline & & D T & 79.78 & 33.36 & 14.97 & 14.15 & 7.84 & 6.14 & 35.37 & 7.34 \\
\hline & & $\mathrm{R}$ & 503.00 & 203.00 & 139.00 & 110.00 & 39.00 & 33.00 & 187.00 & 61.00 \\
\hline & \multirow{4}{*}{$\mathrm{T}$} & $\mathrm{M}$ & 266.55 & 105.21 & 8.11 & 6.34 & 26.50 & 12.38 & 99.61 & 14.10 \\
\hline & & $\mathrm{N}$ & 1017.00 & 1017.00 & 1019.00 & 1008.00 & 1018.00 & 1016.00 & 1006.00 & 1017.00 \\
\hline & & D T & 75.20 & 31.61 & 13.58 & 12.39 & 7.59 & 5.85 & 33.88 & 7.28 \\
\hline & & $\mathrm{R}$ & 555.00 & 203.00 & 139.00 & 110.00 & 47.00 & 33.00 & 188.00 & 65.00 \\
\hline
\end{tabular}

Nota. H: Hombre, M: Mujer, T: Total, R: Rango, TR: total de respuestas, TA: total de aciertos, O: omisiones, C: comisiones, CON: índice de concentración, TR+: línea con mayor $\mathrm{n}^{\mathrm{o}}$ de elementos intentados, TR-: línea con menor $\mathrm{n}^{\mathrm{o}}$ de elementos intentados, VAR: índice de variación o diferencia (TR+) - (TR-).

Excepto en la medida de omisiones, $\left[F_{\text {asintótica }}(5,467.540)=1.41, p=.21\right]$, se encontraron diferencias significativas en todas y cada una de las medidas del $\mathrm{d} 2$, total de respuestas, $\left[F_{\text {asintótica }}(5,466.051)=138.6 ; p<.001, \omega_{\text {estimada }}^{2}=.04\right]$; total de aciertos, $\left[F_{\text {asintótica }}(5,467.825)=132.48 ; p<.001, \omega_{\text {estimada }}^{2}=.39\right]$; comisiones, $\left[F_{\text {asintótica }}(5,456.753)\right.$ $\left.=5.33, p<.001, \omega_{\text {estimada }}^{2}=.02\right]$; línea con mayor número de elementos intentados, $\left[F_{\text {asintótica }}(5,469.855)=37.57, p<.001, \omega_{\text {estimada }}^{2}=.03\right]$; línea con menor número de elementos intentado, $\left[F_{\text {asintótica }}(5,465.128)=110.74 ; p<.001, \omega_{\text {estimada }}^{2}=.09\right]$; efectividad total en la prueba, $\left[F_{\text {asintótica }}(5,460.953)=162.67, p<.001, \omega_{\text {estimada }}^{2}=.43\right]$; índice de concentración, $\left[F_{\text {asintótica }}(5,462.77)=138.14, p<.001, \omega_{\text {estimada }}^{2}=.39\right]$; y el índice de variación o diferencia, $\left[F_{\text {asintótica }}(5,469.21)=2.31, p<.05, \omega_{\text {estimada }}^{2}=.01\right]$. Los análisis de tendencia demostraron la existencia de una relación lineal ascendente en función de la edad para las medidas de total de respuestas, $\left[F_{(1,1012)}=712.5, p<.001\right]$; total de aciertos, $\left[F_{(1,1012)}=672.42, \mathrm{p}<.001\right]$; comisiones, $\left[F_{(1,1003)}=22.65, p<.001\right]$; línea con mayor número de elementos intentados, $\left.\left[F_{(1,1013)}\right)=196.34, p<.001\right]$; línea con menor número de elementos intentados, $\left[F_{(1,1011)}=505.91, p<.001\right]$; efectividad total en la prueba, $\left[F_{(1,1001)}\right.$ $=787.40, p<.001]$; e índice de concentración, $\left[F_{(1,1001)}=673.16, p<.001\right]$. Por último, se encontró una relación cuadrática en el caso de la medida índice de variación o diferencia, 
$\left[F_{(1,1012)}=4.35, p<.05\right]$. Una representación gráfica de las curvas de crecimiento en las medidas de productividad y efectividad se muestra en las figuras 1-4.

Figura 1. Curva de crecimiento con la edad en total de respuestas (TR)

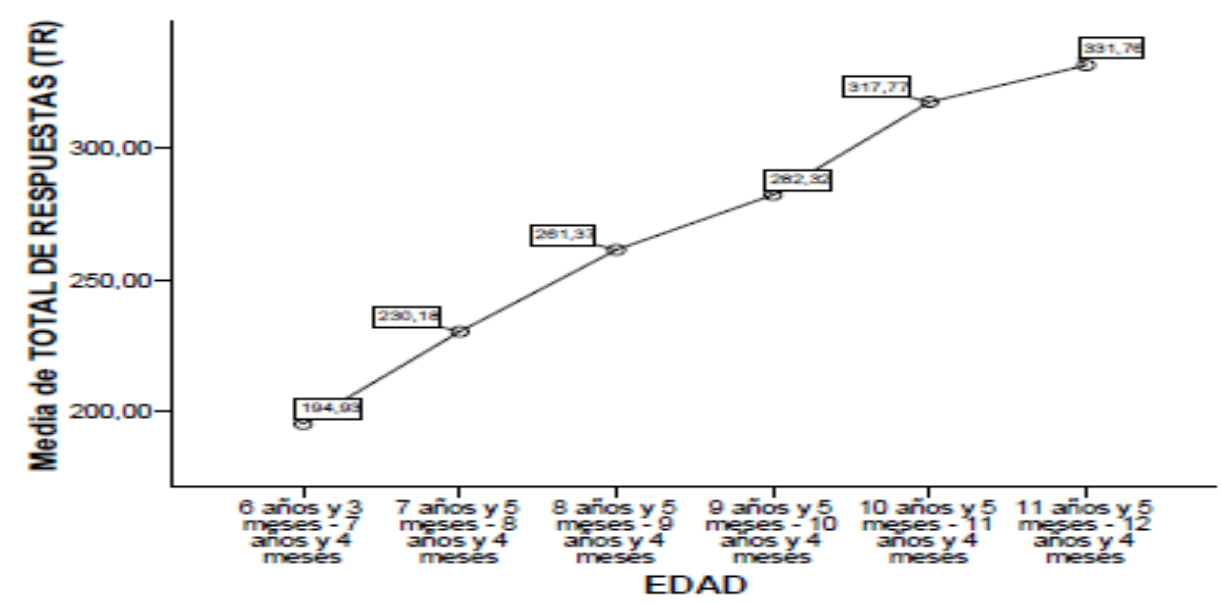

Figura 2. Curva de crecimiento con la edad en total de aciertos (TA)

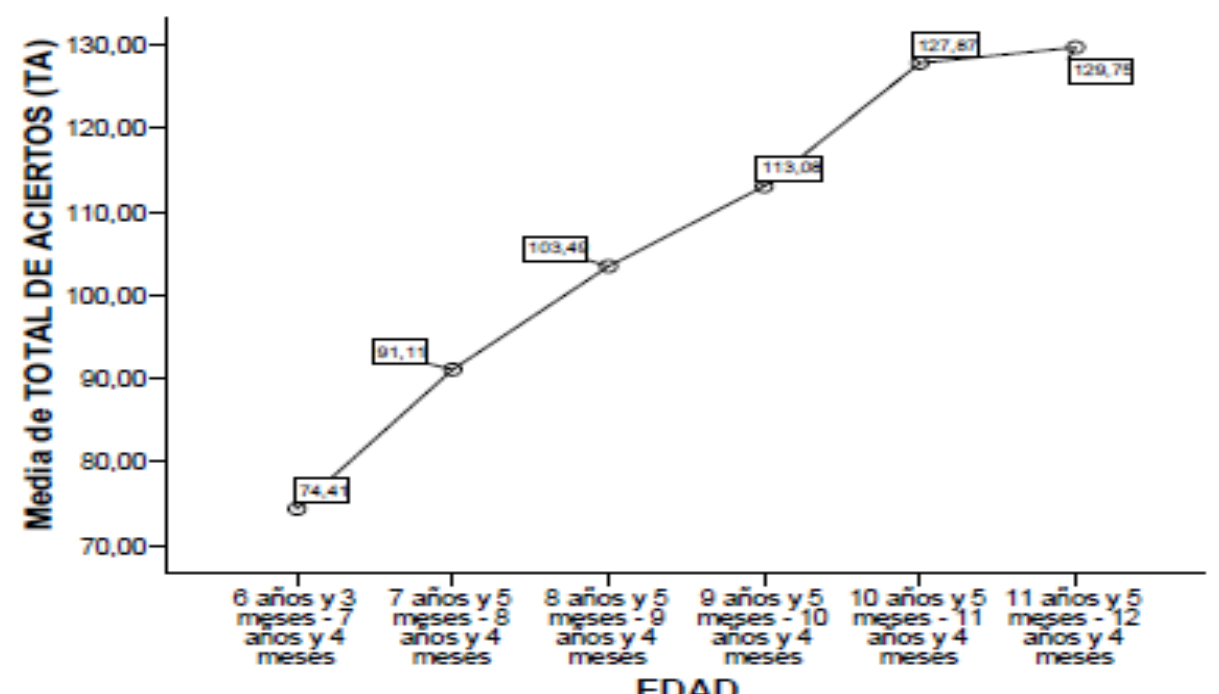

Figura 3. Curva de crecimiento con la edad en efectividad total en la prueba (TOT) 


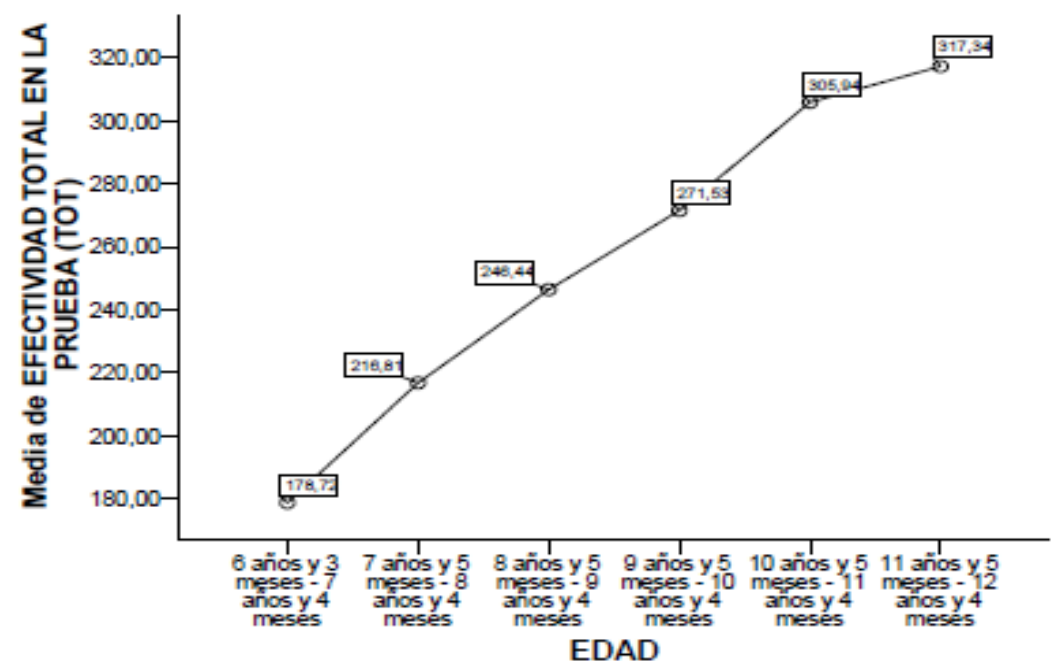

Figura 4. Curva de crecimiento con la edad en concentración (CON)

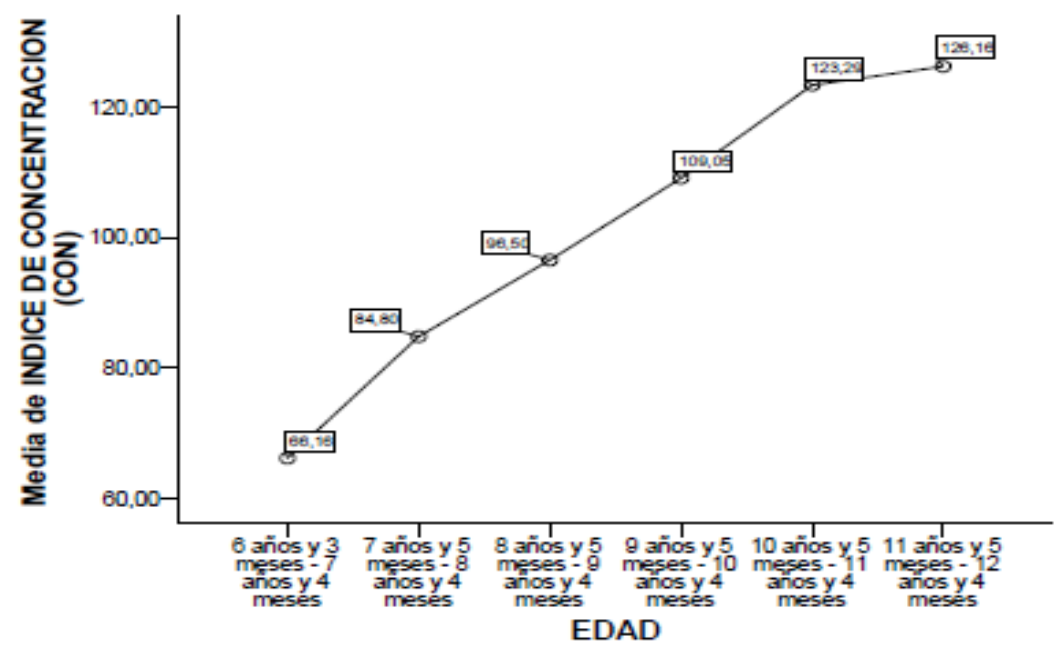

Tras estos resultados llevamos a cabo contrastes a posteriori de las diferencias par a par centrándonos especialmente en las medidas de productividad y efectividad (TR, TA, TOT y CON) cuyos resultados se muestran en la tabla 3. 
Tabla 3. Medias y desviaciones típicas por grupos de edad en cada una de las subtareas, y valores de $t$

\begin{tabular}{|c|c|c|c|c|c|c|c|}
\hline & GRUPOS & M (DT) & Grupo 2 & Grupo 3 & Grupo 4 & Grupo 5 & Grupo 6 \\
\hline \multirow{6}{*}{ TR } & Grupo 1 & $194.5(55.9)$ & $\mathrm{t}(361)=5.80^{* * *}$ & $\mathrm{t}(376)=11.9 * * *$ & $\mathrm{t}(353)=14.1 * * *$ & $\mathrm{t}(360)=20.1 * * *$ & $\mathrm{t}(358)=22.4^{* * * *}$ \\
\hline & Grupo 2 & $230.1(61.5)$ & & $\mathrm{t}(340)=4.99^{* *}$ & $\mathrm{t}(317)=8.07^{* * * *}$ & $\mathrm{t}(324)=13.7^{* * *}$ & $\mathrm{t}(322)=15.8^{* * * *}$ \\
\hline & Grupo 3 & $261.3(52.4)$ & & & $\mathrm{t}(330)=3.31^{*}$ & $\mathrm{t}(337)=9.01 * * *$ & $\mathrm{t}(334)=11.2^{* * * *}$ \\
\hline & Grupo 4 & $282.3(54.2)$ & & & & $\mathrm{t}(316)=5.47^{*}$ & $\mathrm{t}(314)=7.61 * * *$ \\
\hline & Grupo 5 & $317.7(60.3)$ & & & & & $\mathrm{t}(321)=2.18$ \\
\hline & Grupo 6 & $331.7(60.9)$ & & & & & \\
\hline \multirow{6}{*}{ TA } & Grupo 1 & $74.2(25.9)$ & $\mathrm{t}(362)=6.49 * * *$ & $t(377)=11.5^{* * * *}$ & $t(354)=14.8^{* * * *}$ & $\mathrm{t}(360)=20.7 * * *$ & $\mathrm{t}(359)=21.4 * * *$ \\
\hline & Grupo 2 & $91.1(26.0)$ & & $\mathrm{t}(340)=4.67 *$ & $\mathrm{t}(317)=8.02^{* * * *}$ & $\mathrm{t}(323)=13.5^{* * *}$ & $\mathrm{t}(322)=14.2^{* * * *}$ \\
\hline & Grupo 3 & $103.4(23.3)$ & & & $\mathrm{t}(332)=3.57 * *$ & $\mathrm{t}(338)=9.18^{* * *}$ & $\mathrm{t}(337)=9.87 * * * *$ \\
\hline & Grupo 4 & $113.0(23.1)$ & & & & $\mathrm{t}(315)=5.38^{* *}$ & $\mathrm{t}(314)=6.05^{* * * *}$ \\
\hline & Grupo 5 & $127.8(24.3)$ & & & & & $\mathrm{t}(320)=0.69$ \\
\hline & Grupo 6 & $129.7(22.9)$ & & & & & \\
\hline \multirow{6}{*}{ TOT } & Grupo 1 & $178.7(52.2)$ & $\mathrm{t}(355)=6.45^{* * * *}$ & $\mathrm{t}(371)=11.7^{* * * * *}$ & $\mathrm{t}(348)=15.5^{\text {***** }}$ & $\mathrm{t}(356)=21.5^{* * *}$ & $\mathrm{t}(352)=23.3^{* * * *}$ \\
\hline & Grupo 2 & $216.8(61.7)$ & & $\mathrm{t}(337)=4.90^{* * * *}$ & $\mathrm{t}(314)=8.75^{* * * *}$ & $\mathrm{t}(322)=14.4^{* * *}$ & $\mathrm{t}(318)=16.1^{* * * *}$ \\
\hline & Grupo 3 & $246.4(50.8)$ & & & $\mathrm{t}(330)=4.10^{* * *}$ & $\mathrm{t}(338)=9.87^{* * *}$ & $\mathrm{t}(334)=11.6^{* * * *}$ \\
\hline & Grupo 4 & $271.5(55.1)$ & & & & $\mathrm{t}(315)=5.51^{* *}$ & $\mathrm{t}(311)=7.30^{* * * *}$ \\
\hline & Grupo 5 & $305.9(56.9)$ & & & & & $\mathrm{t}(319)=1.83$ \\
\hline & Grupo 6 & $317.3(56.0)$ & & & & & \\
\hline \multirow{6}{*}{$\mathrm{CON}$} & Grupo 1 & $66.1(26.3)$ & $\mathrm{t}(356)=6.70 * * *$ & $\mathrm{t}(372)=11.2 * * *$ & $\mathrm{t}(349)=15.2^{* * * *}$ & $\mathrm{t}(356)=20.5 * * *$ & $\mathrm{t}(353)=21.4^{* * * *}$ \\
\hline & Grupo 2 & $84.8(28.9)$ & & $\mathrm{t}(337)=4.11^{* * * *}$ & $\mathrm{t}(314)=8.23^{* * *}$ & $\mathrm{t}(321)=13.2^{* * *}$ & $\mathrm{t}(318)=14.1^{* * * *}$ \\
\hline & Grupo 3 & $96.5(26.0)$ & & & $\mathrm{t}(330)=4.35^{* * *}$ & $\mathrm{t}(337)=9.41^{* * *}$ & $\mathrm{t}(334)=10.3^{* * *}$ \\
\hline & Grupo 4 & $109.0(24.3)$ & & & & $\mathrm{t}(314)=4.83^{* *}$ & $\mathrm{t}(311)=5.78^{* *}$ \\
\hline & Grupo 5 & $123.2(25.0)$ & & & & & $\mathrm{t}(318)=0.98$ \\
\hline & Grupo 6 & $126.1(25.7)$ & & & & & \\
\hline
\end{tabular}

Los resultados mostraron que se producen cambios significativos $y$ sistemáticos en las variables que miden productividad y efectividad, ya que se observa una tendencia lineal significativa y existen diferencias significativas entre los diferentes grupos de edad establecidos, con la excepción de los grupos 10.5-11.4 y 11.5-12.4 que no se diferencian entre sí en los contrastes a posteriori par a par.

\section{B. Estudio de baremación}

El sumatorio de las puntuaciones obtenidas por cada rango de edad para cada una de las variables del d2 fue convertido en percentiles con el propósito de establecer una escala de los valores de las puntuaciones directas de cada grupo de edad que representan el percentil 75, 50 y 25 . Los resultados encontrados pueden consultarse en la tabla 4.

En cada una de las tablas, las columnas centrales contienen los valores de las puntaciones directas de las nueve variables empleadas en la tipificación (TR a VAR), en la primera, a la izquierda, están las puntuaciones centiles (Pc) correspondientes a las puntuaciones directas de las columnas centrales. Una vez obtenida la puntuación directa de un sujeto mediante el uso del ejemplar autocorregible en una variable, se entra en el cuerpo de la tabla por la columna central de esa puntuación hasta encontrar el valor directo, y a la misma altura se hallan las correspondientes puntación centil (Pc en la primera columna de la izquierda). Este proceso hay que repetirlo con todas las puntuaciones directas que se desee interpretar. 
JIMÉNEZ et al. Test de atención D2: datos normativos y desarrollo evolutivo

Tabla 4. Baremos en varones y mujeres de 6 a 12 años en las medidas del d2

Baremos en varones y mujeres de 6 años y 3 meses - 7 años y 4 meses

\begin{tabular}{|c|c|c|c|c|c|c|c|c|c|c|}
\hline & & TR & TA & $\mathrm{O}$ & C & TOT & $\mathrm{CON}$ & TR+ & TR- & VAR \\
\hline \multirow{2}{*}{$\mathrm{N}$} & Válidos & 200 & 201 & 201 & 196 & 195 & 196 & 200 & 199 & 199 \\
\hline & Perdidos & 5 & 4 & 4 & 9 & 10 & 9 & 5 & 6 & 6 \\
\hline \multirow{3}{*}{ Percentiles } & 25 & 162 & 60 & 1 & 1 & 148 & 50 & 16 & 5 & 10 \\
\hline & 50 & 192 & 75 & 4 & 3 & 177 & 68 & 21 & 7 & 13 \\
\hline & 75 & 227 & 89 & 10 & 9 & 210 & 84 & 26 & 9 & 18 \\
\hline
\end{tabular}

Baremos en varones y mujeres de 7 años y 5 meses - 8 años y 4 meses

\begin{tabular}{|c|c|c|c|c|c|c|c|c|c|c|}
\hline & & TR & TA & $\mathrm{O}$ & $\mathrm{C}$ & TOT & $\mathrm{CON}$ & TR+ & TR- & VAR \\
\hline \multirow{2}{*}{$\mathrm{N}$} & Válidos & 163 & 163 & 164 & 162 & 161 & 161 & 164 & 164 & 164 \\
\hline & Perdidos & 4 & 4 & 3 & 5 & 6 & 6 & 3 & 3 & 3 \\
\hline \multirow{3}{*}{ Percentiles } & 25 & 192 & 75 & 1 & 2 & 179 & 66 & 19 & 6 & 9 \\
\hline & 50 & 227 & 93 & 3 & 4 & 215 & 89 & 23 & 11 & 12 \\
\hline & 75 & 266 & 108 & 6 & 8 & 254 & 104 & 27 & 14 & 18 \\
\hline
\end{tabular}

Baremos en varones y mujeres de 8 años y 5 meses - 9 años y 4 meses

\begin{tabular}{|c|c|c|c|c|c|c|c|c|c|c|}
\hline & & TR & TA & $\mathrm{O}$ & $\mathrm{C}$ & TOT & $\mathrm{CON}$ & $\mathrm{TR}+$ & TR- & VAR \\
\hline \multirow{2}{*}{$\mathrm{N}$} & Válidos & 178 & 178 & 178 & 177 & 177 & 177 & 178 & 178 & 178 \\
\hline & Perdidos & 1 & 1 & 1 & 2 & 2 & 2 & 1 & 1 & 1 \\
\hline \multirow{3}{*}{ Percentiles } & 25 & 225 & 92 & 2 & 1 & 216 & 83 & 21 & 9 & 10 \\
\hline & 50 & 259 & 104 & 4 & 3 & 249 & 100 & 24 & 13 & 12 \\
\hline & 75 & 294 & 117 & 9 & 7 & 277 & 113 & 29 & 15 & 16 \\
\hline
\end{tabular}

Baremos en varones y mujeres de 9 años y 5 meses - 10 años y 4 meses

\begin{tabular}{|c|c|c|c|c|c|c|c|c|c|c|}
\hline & & TR & TA & $\mathrm{O}$ & $\mathrm{C}$ & TOT & $\mathrm{CON}$ & TR+ & TR- & VAR \\
\hline \multirow{2}{*}{$\mathrm{N}$} & Válidos & 155 & 155 & 155 & 154 & 154 & 154 & 155 & 155 & 155 \\
\hline & Perdidos & 1 & 1 & 1 & 2 & 2 & 2 & 1 & 1 & 1 \\
\hline \multirow{3}{*}{ Percentiles } & 25 & 247 & 102 & 1 & 1 & 237 & 96 & 23 & 12 & 9 \\
\hline & 50 & 283 & 115 & 3 & 3 & 270 & 111 & 26 & 14 & 13 \\
\hline & 75 & 318 & 129 & 7 & 6 & 307 & 126 & 31 & 16 & 17 \\
\hline
\end{tabular}

Baremos en varones y mujeres de 10 años y 5 meses - 11 años y 4 meses

\begin{tabular}{|c|c|c|c|c|c|c|c|c|c|c|}
\hline & & TR & $\mathrm{TA}$ & $\mathrm{O}$ & $\mathrm{C}$ & TOT & $\mathrm{CON}$ & TR+ & TR- & VAR \\
\hline \multirow{2}{*}{$\mathrm{N}$} & Válidos & 162 & 161 & 162 & 162 & 162 & 161 & 162 & 161 & 162 \\
\hline & Perdidos & 2 & 3 & 2 & 2 & 2 & 3 & 2 & 3 & 2 \\
\hline \multirow{3}{*}{ Percentiles } & 25 & 281 & 113 & 2 & 0 & 270 & 107 & 26 & 14 & 10 \\
\hline & 50 & 319 & 128 & 4 & 2 & 309 & 124 & 29 & 15 & 12 \\
\hline & 75 & 361 & 144 & 7 & 5 & 344 & 141 & 33 & 19 & 15 \\
\hline
\end{tabular}

Baremos en varones y mujeres de 11 años y 5 meses - 12 años y 4 meses

\begin{tabular}{|c|c|c|c|c|c|c|c|c|c|c|}
\hline & & TR & TA & $\mathrm{O}$ & $\mathrm{C}$ & TOT & $\mathrm{CON}$ & TR+ & TR- & VAR \\
\hline \multirow{2}{*}{$\mathrm{N}$} & Válidos & 160 & 160 & 160 & 158 & 158 & 158 & 160 & 160 & 160 \\
\hline & Perdidos & 2 & 2 & 2 & 4 & 4 & 4 & 2 & 2 & 2 \\
\hline \multirow{3}{*}{ Percentiles } & 25 & 289 & 117 & 2 & 0 & 281 & 113 & 27 & 13 & 10 \\
\hline & 50 & 328 & 130 & 6 & 2 & 321 & 128 & 30 & 16 & 13 \\
\hline & 75 & 367 & 145 & 11 & 5 & 353 & 143 & 34 & 19 & 18 \\
\hline
\end{tabular}

Nota. TR: total de respuestas, TA: total de aciertos, O: omisiones, C: comisiones, TOT: efectividad total en la prueba, CON: índice de concentración, TR+: línea con mayor $\mathrm{n}^{\circ}$ de elementos intentados, TR-: línea con menor $\mathrm{n}^{\mathrm{o}}$ de elementos intentados, VAR: índice de variación o diferencia (TR+) - (TR-).

\section{DISCUSIÓN}

En el presente estudio hemos analizado el desarrollo evolutivo de la atención en población escolar de educación primaria que no presenta sospecha de TDAH, a partir de las medidas y parámetros que establece el Test de atención d2. En líneas generales se 
ha observado que el rendimiento mejora sustancialmente en función de la edad cuando hemos analizado las medidas de productividad y efectividad, esto es, el número total de elementos procesados o intentados en todo el test (TR); el número total de aciertos (TA); el número de elementos procesados (TR) menos el número total de errores cometidos (TOT); y el número de elementos relevantes correctamente marcados (TA) menos el número de comisiones (CON). En todas estas medidas hemos observado una tendencia lineal significativa pero en los contrastes a posteriori de las diferencias par a par, hemos encontrado que este crecimiento se observa hasta la edad de 10 años y cinco meses, ya que luego no hemos encontrado diferencias significativas entre este rango de edad y el inmediatamente posterior de 11 años y cinco meses. En general, los estudios sobre el desarrollo de la atención a nivel selectivo han puesto de relieve que los oscilamientos de la atención son más lentos a lo largo de toda la infancia que en la edad adulta. Esta mayor lentitud va disminuyendo progresivamente, de forma que los niños de 7 años cuando se enfrentan a tareas de escucha dicótica suelen ser más lentos a la hora de reorientar su atención de un oído a otro, si bien a los 11 años la mayoría de los niños son capaces de desplazar su foco de atención de un mensaje a otro casi con la misma rapidez que los adultos (García, 2008). También se ha observado que los niños entre 5 y 7 años de edad tienen más dificultad para ignorar las dimensiones irrelevantes que niños más mayores, pero según evolucionan se ven menos afectados por la presencia de estímulos distractores. En el presente estudio hemos observado precisamente que a partir de la edad de 6 años y 3 meses se produce una mejora sustancial en la velocidad de procesamiento, en la cantidad de trabajo realizado y en la motivación, y una cierta estabilidad cuando nos aproximamos a los 11 años de edad.

No obstante, Konrad et al. (2005) mediante técnicas de resonancia magnética funcional analizaron los circuitos neuronales asociados a distintos subprocesos de la atención como el nivel de alertamiento y la capacidad que presentan los niños para mantenerse en ese estado conforme transcurre el tiempo, la capacidad para orientar y cambiar el foco de atención, así como el control ejecutivo de la atención en niños de 8 a 12 años de edad y en adultos. Los niños mostraron en general un nivel de alertamiento menor y un efecto mayor de reorientación y de interferencia respecto al control ejecutivo de la atención. Asimismo, los niños mostraban de manera significativa una reducción de la activación cerebral en aquellas regiones que se ubican en el lado derecho del cerebro en la zona medio-frontal implicada en la fase de alertamiento, en la unión temporoparietal del lado derecho implicada en la fase de atención espacial, y en la corteza prefrontal dorsolateral implicada en el control ejecutivo de la atención. En cambio, los niños llegaron a activar muchas más regiones del cerebro que se encuentran fuera de las zonas que tendrían mayor implicación en estos procesos, como por ejemplo el gyrus frontal superior durante la atención espacial y el gyrus temporal superior durante el control ejecutivo de la atención. Los datos sugieren que hay una transición entre los 
sistemas funcionales aún inmaduros que sirven de soporte a estos subprocesos de la atención en niños, a redes o circuitos más definidos en adultos y que estas diferencias pueden estar reflejando cambios evolutivos en estrategias cognitivas y a nivel morfológico.

Aunque nuestra muestra de estudio está constituida por población que no presenta sospecha de TDAH, sin embargo, estudios como el realizado por Morales y Meneses (2003) encontraron que los niños con TDAH presentaban dificultades en mantener su atención a lo largo del tiempo, exhibían alteraciones en la habilidad de seleccionar un estímulo blanco en un campo visual, cuando su atención es controlada por pistas centrales, y presentaban alteraciones en los procesos de atención enfocada y en las funciones ejecutivas, pero mantenían íntegros los procesos de atención viso-espacial. Estos mismos autores concluían también que si el componente de alertamiento depende de la integridad de la formación reticular, de los núcleos intralaminares del tálamo y de la corteza frontal, como ha sido sugerido por Posner y Petersen (1990). Por lo tanto, los individuos con TDAH presentan alteraciones en alguna de estas regiones o en las vías responsables de su activación sostenida. En el caso de las alteraciones observadas en la atención dividida y en las funciones ejecutivas evaluadas mediante la prueba Stroop y la prueba de categorización de Wisconsin, se piensa que están asociadas con disfunciones en la corteza frontal. En cambio, los procesos de atención viso-especial que dependen del sistema de atención posterior, que incluye al colículo superior, el núcleo pulvinar del tálamo y la corteza parietal (Posner y Petersen, 1990) se encontrarían conservados en niños con TDAH.

\section{Agradecimientos}

Esta investigación ha sido financiada por la Agencia Canaria de Investigación, Innovación y Sociedad de la Información de la Consejería de Educación, Universidades y Sostenibilidad del Gobierno de Canarias, con ref. PI 2007/012 del que es IP el primer autor.

\section{REFERENCIAS}

Barkley, R.A. (2006). Attention-deficit hyperactivity disorder: a handbook for diagnosis and treatment. Nueva York: Guilford Press.

Brickenkamp, R. (2002). D2, Test de atención (trad. al castellano por n. Seisdedos). Madrid: TEA Ediciones.

Castillo, M.D. (2009). La atención. Madrid: Pirámide.

Cuesta, M., de Iscar, M.J., Begega, M.A., Méndez, M., Álvarez, L., Solís, G., Fernández, B. y Arias, J.L. (2007). Psychometric properties of the $\mathrm{d} 2$ selective attention test in a sample of premature and born-at-term babies. Psicothema, 19, 706-710.

García, J. (2008). Psicología de la atención. Madrid: Síntesis. 
Jasper, H.H. y Bertrand, G. (1966). Thalamic units involved in somatic sensation and voluntary and involuntary movements in man. En D.P. Purpura y M.D. Yahr (Eds.), The Thalamus (pp. 365-390). Nueva York: Columbia University Press.

Konrad, K., Neufang, S., Thiel, C.M., Specht, K., Hanisch, C., Fan, J., Herpertz-Dahlmann, B. y Knk, G.R. (2005). Development of attentional networks: An fMRI study with children and adults. Neuroimage, 28, 429-439.

Luria, A.R. (1984). El cerebro en acción. Barcelona: Martínez Roca.

Miranda, A., García, R. y Soriano, M. (2005). Habilidad narrativa de los niños con trastorno por déficit de atención con hiperactividad. Psicothema, 17, 227-232.

Morales, G. y Meneses, S. (2003). Evaluación de procesos atencionales y funciones ejecutivas en niños con trastorno de la atención con hiperactividad. Revista Neuropsicología, Neuropsiquiatría y Neurociencias, 5, 138-158.

Parasuranam, R. (1984). Sustained attention in detection and discrimination. En R. Parasuranam y D.R. Davies (Eds.), Varieties of Attention (pp. 243-271). Nueva York: Academic Press.

Posner, M. y Petersen, S. (1990). The attentional system of the human brain. Annual Review Neuroscience, 13, 25-34.

Rafal, R.D. y Posner, M.I. (1987). Deficits in visual spatian attentional following thalamic lesions. Proceedings of the National Academy of Science, 84, 7349-7353.

Rebollo, M.A. y Montiel, S. (2006). Atención y funciones ejecutivas. Revista de Neurología, 42, 3-7.

Vega, M. (1984). Introducción a la psicología cognitiva. Madrid: Alianza Editorial.

Zillmer, E.A. y Spiers, M.V. (1998). Principles of clinical neuropsychology. Pacific Groove, CA: Brooks/Cole. 